SHEP 90/91-29

NBI-HE-91-27

\title{
Stochastic Quantization vs. KdV Flows in 2D Quantum Gravity
}

\author{
J. Ambjørn, ${ }^{1}$ C.V. Johnson ${ }^{2}$ and T.R. Morris ${ }^{2}$ \\ ${ }^{1}$ Niels Bohr Institute \\ Blegdamsvej 17, DK-2100 Copenhagen Ø, Denmark \\ and \\ ${ }^{2}$ Physics Department \\ The University of Southampton \\ SO9 5NH, U.K.
}

\begin{abstract}
We consider the stochastic quantization scheme for a non-perturbative stabilization of $2 \mathrm{D}$ quantum gravity and prove that it does not satisfy the $\mathrm{KdV}$ flow equations. It therefore differs from a recently suggested matrix model which allows real solutions to the KdV equations. The behaviour of the Fermi energy, the free energy and macroscopic loops in the stochastic quantization scheme are elucidated.
\end{abstract}




\section{Introduction}

There are now at least two proposals for a non-perturbatively stable formulation of two dimensional quantum gravity. On the one hand there is the "5th time" method[1] rediscovered in the context of Parisi-Sourlas reduction of a one dimensional supersymmetric matrix model[2] a.k.a. stochastic quantization ${ }^{1}[4][5][6][7]$

and on the other hand a definition[8][9] following from non-perturbative KdV flows[10][11]. Both proposals agree[8][2] with the perturbative (i.e. genus by genus) results from hermitian matrix models[12] as indeed they must, while also providing (apparently) a physically sensible non-perturbative extension.

By "physically sensible" we mean at least that the string susceptibility and correlations of all local operators are real and free of singularities (on the real axis). This has been shown to be true in the KdV flow method[8][9], and in the stochastic quantization method can be expected from the construction; Recall that it is not true of the full non-perturbative result derived from hermitian matrix models [13][14]. We also require that macroscopic loop expectation values be physically sensible. For the KdV flow method this has been checked in ref.[9]. Part of the purpose of this paper will be to demonstrate similarly sensible results for the stochastic quantization method. ${ }^{2}$

Naïvely one might expect that 2D quantum gravity has a unique nonperturbative extension, and hence that the two proposals are equivalent. This is not the case. We demonstrate this below, both numerically and analytically. It follows unfortunately that at present the question of the "correct" non-perturbative extension becomes a philosophical one. The problem is of course that no general principle of non-perturbative gravity has yet been defined, and since both methods have the same perturbative expansion, it is hard to promote one method over another if they have no obvious flaws.

The proof that the KdV flows are violated non-perturbatively in the stochastic quantization scheme will be as follows. The KdV flows induced by the operators

\footnotetext{
1 Other suggestions based on stochastic quantization are to be found in ref.[3].

2 Our interest in this problem was triggered by some remarks in ref.[15] and it seems that our conclusions differ from the the ones presented there.
} 
$\mathcal{O}_{k}$ are given by[11]:

$$
\frac{\partial \rho}{\partial t_{k}}=R_{k+1}^{\prime}[\rho]
$$

Here the $t_{k}$ 's are couplings conjugate to the operators $\mathcal{O}_{k}$ and $\rho$ is the string susceptibility related to the free energy $\Gamma$ by $\Gamma^{\prime \prime}=-\rho . \quad \Gamma$ is given in terms of the partition function as $Z=\exp (\Gamma)$. Primes refer to differentiation with respect to $\mu$, the cosmological constant. (The string coupling has been absorbed into $\mu$ and the couplings $t_{k}$ ). The $R_{k}$ 's are the Gelfand-Dikii differential polynomials[16]

If we assume that no further dimensionful parameter arises at the nonperturbative level, then these equations (1.1) are satisfied non-perturbatively if and only if $\rho$ satisfies the string eqn.[9]:

$$
0=\rho \mathcal{R}^{2}-\frac{1}{2} \mathcal{R} \mathcal{R}^{\prime \prime}+\frac{1}{4}\left(\mathcal{R}^{\prime}\right)^{2}
$$

where for the full massive theory

$$
\mathcal{R}=\sum_{k=0}^{\infty}\left(k+\frac{1}{2}\right) t_{k} R_{k}[\rho]-\mu .
$$

For the purposes of this paper however, we will only be interested in pure gravity in which case, without loss of generality, we take only $t_{2} \neq 0$ and

$$
\mathcal{R}=\rho^{2}-\rho^{\prime \prime} / 3-\mu
$$

Now for negative cosmological constant $\mu$, if $\rho$ satisfies eqn. (1.2) and has an asymptotic expansion then it follows from (1.4) and (1.2) that the spherical contribution $^{3}$ must vanish $\rho_{0}=0$ or be imaginary $\rho_{0}= \pm i|\mu|^{1 / 2}$. (These are the roots of the algebraic equation obtained by deleting all derivative terms in (1.2)). For negative $\mu$ an asymptotic expansion for $\rho$ does indeed exist in the supersymmetric 1D matrix model since this expansion just corresponds to the WKB expansion. Because it is real the leading term is of the form $\rho_{0}=\kappa \sqrt{|\mu|}$ for some real constant $\kappa$, by scaling arguments. We will prove that $\kappa$ is different from zero. It follows that the string susceptibility calculated in the stochastic quantization method cannot satisfy (1.2) and hence violates eqns. (1.1) non-perturbatively.

\footnotetext{
3 up to analytic terms in $\mu$ which are always discarded
} 
We note in passing that it is in fact natural to introduce a new non-perturbative parameter $\sigma$ into (1.2) corresponding to the position of the eigenvalue space boundary[17]. It corresponds physically to a world-sheet boundary cosmological constant. In this case $(1.2)$ takes the form $0=(\rho-\sigma) \mathcal{R}^{2}-\frac{1}{2} \mathcal{R} \mathcal{R}^{\prime \prime}+\frac{1}{4}\left(\mathcal{R}^{\prime}\right)^{2}$. It is equivalent to $(1.2)$ under the finite 'gauge' transformation $\exp \left\{-\sigma L_{-1}\right\}$ and so does not alter our conclusions. $\left(\epsilon L_{-1}\right.$ generates the $\mathrm{KdV}$ galilean transformation: $\left.\delta t_{k}=\epsilon(k+3 / 2) t_{k+1}, \quad \delta \rho=\delta \sigma=\epsilon\right)$.

It may seem a touch paradoxical that non-perturbative information can be deduced from perturbative arguments in the regime $\mu<0$. We would therefore like to emphasise that the asymptotic results for $\mu<0$ in both schemes should properly be regarded as non-perturbative. From the physical point of view both schemes derive their connection to pure $2 \mathrm{D}$ quantum gravity/0D string theory indirectly through exact agreement with the $\mu>0$ hermitian matrix model perturbative results, the latter having a direct representation in terms of regularised surfaces through the dual of Feynman diagrams. Neither scheme has a direct worldsheet interpretation. (This is discussed further for the complex matrix model representation of the $\mathrm{KdV}$ flow method in ref.[8]). When $\mu<0$ both schemes lose their interpretation in terms of surfaces since, if for no other reason, they now disagree with the hermitian matrix model. Actually the hermitian matrix model has no interpretation in terms of surfaces here either because the $\mu<0$ region is outside the radius of convergence of its large $N$ Feynman diagram expansion. Indeed mathematically the perturbative results for $\mu<0$ are separated from those for $\mu>0$ by non-smooth behaviour at $\mu=0$. The spherical free energy suffers a 3rd order phase transition; In the stochastic quantization scheme for $\mu>0$ supersymmetry is preserved to all orders of perturbation theory, while for $\mu<0$ supersymmetry is broken even on the sphere. In the representation of the $\mathrm{KdV}$ flow scheme in terms of an eigenvalue spectrum $\lambda \in \mathbb{R}_{+}$the classical eigenvalue distribution hits the "wall" at $\lambda=0$ as $\mu \rightarrow 0^{+}[8][9]$ (more generally[17] $\lambda=\sigma$ as $\mu \rightarrow\left(\sigma^{2}\right)^{+}$).

The rest of this paper is organised as follows: Section 2 derives the spherical string susceptibility for $\mu<0$ by calculating the one point function. The role of the Fermi energy and the free energy in the supersymmetric matrix model is clarified. Macroscopic loops on the sphere are discussed in section 3 and a non-polynomial 
0D hermitian matrix model potential that produces the same results is derived. A numerical comparison of the one-point function in both schemes is made in sect. 4, and in sect. 5 we draw our conclusions.

\section{The Sphere for Negative Cosmological Constant.}

Recall that the stochastic quantization scheme for the simplest matrix models with cubic potentials results in replacing expectation values defined in the hermitian matrix model

$$
<\mathcal{O}(\phi)>=\frac{1}{Z} \int d \phi \mathcal{O}(\phi) \exp (-N \operatorname{Tr} V(\phi))
$$

where $\phi$ is an $N \times N$ hermitian matrix, with expectation values of the same operator in the ground state of $N$ non-interacting fermions which have the single particle Fokker-Planck (FP) hamiltonian

$$
H_{F P}=N\left[-\frac{1}{N^{2}} \frac{d^{2}}{d \lambda^{2}}+V_{F P}(\lambda)\right]
$$

where

$$
V_{F P}(\lambda)=\frac{1}{4}\left(V^{\prime}(\lambda)\right)^{2}-\frac{1}{2} V^{\prime \prime}(\lambda)
$$

Whereas the simplest potentials $V$ that give ordinary $k=2$ critical behaviour are unbounded from below, the Fokker-Planck potential $V_{F P}$ is not, and this is the reason the method leads to non-perturbative stabilization. In fact the expectation value of any observable, which in the hermitian matrix model will be given by (2.1) , is here defined by

$$
<\mathcal{O}(\phi)>_{F P}=\int d \phi \Psi_{0}^{2}(\phi) \mathcal{O}(\phi)
$$

where $\Psi_{0}(\phi)$ is the groundstate wavefunction of the $N$-particle fermionic system. In the following we will restrict ourselves to the spherical approximation and to observables of the form $\mathcal{O}(\phi)=\operatorname{Tr} f(\phi)$. The spherical approximation is in the hamiltonian language equivalent to the WKB approximation and the expectation value of the observables $\operatorname{Tr} f(\phi)$ will be given by:

$$
\frac{1}{N}<\operatorname{Tr} f(\phi)>=\frac{1}{\pi} \int_{\lambda_{l}}^{\lambda_{r}} d \lambda \sqrt{E_{F}-V_{F P}(\lambda)} f(\lambda)
$$


Here $\lambda_{r}$ and $\lambda_{l}$ denote the left- and right turning points, and $E_{F}$ denotes the Fermi energy of the system, i.e. the $N^{\prime}$ th energy level, scaled by $N^{-1}$. The WKB condition for the $N^{\prime}$ th energy level of the hamiltonian (2.2) is

$$
1=\frac{1}{\pi} \int_{\lambda_{l}}^{\lambda_{r}} d \lambda \sqrt{E_{F}-V_{F P}(\lambda)}
$$

To be explicit let us consider the simplest potential $V$ in the hermitian matrix model which leads to $k=2$ critical behaviour:

$$
V(\phi)=g \phi-\phi^{3} / 3
$$

The corresponding FP-potential is

$$
V_{F P}(\lambda ; g)=\frac{1}{4}\left(g-\lambda^{2}\right)^{2}+\lambda
$$

The critical coupling in the matrix model is $g_{c}=3 / 2^{2 / 3}$ and the endpoint of the eigenvalues distribution is $\lambda_{c}=\sqrt{g_{c} / 3}=1 / 2^{1 / 3}$ where $V\left(\lambda_{c} ; g_{c}\right)=g_{c}^{2} / 3=3 \lambda_{c}^{4}$ and the derivatives with respect to $\lambda$ satisfy: $V^{\prime}\left(\lambda_{c},: g_{c}\right)=V^{\prime \prime}\left(\lambda_{c} ; g_{c}\right)=0$.

If we introduce the scaled variables:

$$
g-g_{c}=g_{c} a^{2} \mu, \quad \lambda-\lambda_{c}=\lambda_{c} a y, \quad a=1 / N^{2 / 5}
$$

we can write

$$
\begin{aligned}
V_{F P}(y ; \mu) & =V_{F P}(0 ; \mu)+\lambda_{c}^{4} a^{3} v_{f p}(y ; \mu) \\
v_{f p}(y ; \mu) & =-3 \mu y+y^{3}+a\left[-\frac{3}{2} \mu y^{2}+\frac{1}{4} y^{4}\right]
\end{aligned}
$$

where $V_{F P}(0 ; \mu)$ is a second order polynomial in $g-g_{c}=g_{c} a^{2} \mu$. As in [4] the FP-hamiltonian can be written

$$
\begin{aligned}
H_{F P} & =N\left[V_{F P}(0 ; \mu)+\lambda_{c}^{4} a^{3} h_{f p}\right] \\
h_{f p} & =-4 \frac{d^{2}}{d y^{2}}-v_{f p}(y ; \mu)
\end{aligned}
$$

and if we introduce the scaled Fermi energy $e_{f}$ by

$$
E_{F}=V_{F P}(0 ; \mu)+\lambda_{c}^{4} a^{3} e_{f}
$$


the equation (2.6) for the Fermi energy can be written as

$$
N=\frac{1}{2 \pi} \int_{y_{l}}^{y_{r}} d y \sqrt{e_{f}-v_{f p}(y ; \mu)}
$$

While the original hermitian matrix model even at the spherical level is defined only for $g>g_{c}$, i.e. for $\mu>0$, the FP-potential is defined for all real $\mu$. Since $E_{F}$ in the spherical limit can be a function of the coupling constant $g$ only (i.e. $g_{c}$ and $\left.g-g_{c}\right)$, we conclude that

$$
e_{f}=|\mu|^{3 / 2} f^{( \pm)}(a \sqrt{|\mu|})
$$

where $f^{( \pm)}(\cdot)$ might be different functions for $\mu>0$ and $\mu<0$. Let us assume that the $f$ 's have the following expansions

$$
f^{( \pm)}(x)=f_{0}^{( \pm)}+O(x)
$$

where the constants $f_{0}^{( \pm)}$are to be determined from (2.11). By differentiating (2.11) with respect to $|\mu|$ and taking the limit $a \rightarrow 0$ one gets the following equations for $f_{0}^{( \pm)}$:

$$
0=\lim _{a \rightarrow 0} \int_{y_{l}}^{y_{r}} d y \frac{\frac{1}{2} \sqrt{|\mu|} f_{0}^{( \pm)} \pm\left(y+\frac{1}{2} a y^{2}\right)}{\sqrt{f_{0}^{( \pm)}|\mu|^{3 / 2}-v_{f p}(y ; \mu)}}
$$

The integration limits, the turning points, before taking $a \rightarrow 0$ are given by

$$
y_{r}=\sqrt{|\mu|} z_{r}\left(f_{0}^{( \pm)}\right)+O(a), \quad y_{l}=-\frac{4}{a}-\frac{3}{4} \mu a+O\left(a^{2}\right)
$$

Here $\sqrt{|\mu|} z_{r}$ denotes the right turning point solution in the limit $a \rightarrow 0$. We have

$$
f_{0}^{( \pm)} \pm 3 z_{r}-z_{r}^{3}=0
$$

Subtracting from $\pm(2.14)$ the same equation with $\mu=0$, and then changing variables as $y \rightarrow \sqrt{|\mu|} z$ we obtain the convergent integrals:

$$
\int_{-\infty}^{z_{r}} d z \frac{\frac{1}{2} f_{0}^{( \pm)}}{\sqrt{f_{0}^{( \pm)} \pm 3 z-z^{3}}}=\mp \int_{-\infty}^{z_{r}} d z\left[\frac{z}{\sqrt{f_{0}^{( \pm)} \pm 3 z-z^{3}}}-\frac{z}{\sqrt{|z|^{3}}}\right] \pm \int_{z_{r}}^{0} d z \frac{z}{\sqrt{|z|^{3}}}
$$


The solution of (2.17) is well known for $\mu>0$ due to the inherent supersymmetry of the FP-hamiltonian [2], which is unbroken for $\mu>0$, and is given by

$$
f_{0}^{(+)}=-2 \quad\left(\text { and } \quad z_{r}=-2\right)
$$

For $\mu<0$ it is readily seen that $f_{0}^{(-)}>0$ and $z_{r}>0$, but the integrals can not be expressed as elementary integrals. Numerical integration gives

$$
f_{0}^{(-)} \approx 8.0 \quad\left(\text { and } \quad z_{r} \approx 1.52\right)
$$

Let us now turn to the observables of the theory. The simplest and most fundamental ones in the hermitian matrix model are the puncture operator and the susceptibility. In the case of the potential (2.7) the 'bare' puncture operator and the susceptibitity can be defined as follows

$$
\begin{gathered}
<\mathcal{O}_{0}>=-\frac{1}{N^{2}} \Gamma^{\prime}(g)=<\frac{1}{N} \operatorname{Tr} \phi>. \\
\rho(g)=-\frac{d<\mathcal{O}_{0}>}{d g}=\frac{1}{N^{2}} \Gamma^{\prime \prime}(g)
\end{gathered}
$$

In (2.20) $\Gamma$ denotes the free energy of the hermitian matrix model. The FP-version of (2.20) can be found from (2.4), or in the spherical approximation from (2.5)

$$
<\mathcal{O}_{0}>_{F P}=\frac{1}{\pi} \int_{\lambda_{l}}^{\lambda_{r}} d \lambda \lambda \sqrt{E_{F}-V_{F P}(\lambda)}=\lambda_{c}\left(1+\frac{a^{7 / 2}}{2 \pi} \int_{y_{l}}^{y_{r}} d y y \sqrt{e_{f}-v_{f p}(y)}\right) .
$$

and we get

$$
\rho_{F P}(\mu)=\frac{\lambda_{c}}{g_{c}} \frac{a^{3 / 2}}{4 \pi} \int_{y_{l}}^{y_{r}} d y \frac{y\left(e_{f}^{\prime}+3\left(y+\frac{1}{2} a y^{2}\right)\right)}{\sqrt{e_{f}-v_{f p}(y)}}
$$

Inserting the integration limits (2.15) and subtracting, as in (2.17), the expression for $\mu=0$, leads to the following result :

$$
\rho_{F P}(\mu)-\rho_{F P}(0)=\frac{\lambda_{c}}{2 g_{c}}\left(c_{1} e_{f}^{\prime}(\mu) a+O\left((\sqrt{|\mu|} a)^{3 / 2}\right)\right)
$$

where

$$
c_{1}=\frac{\sqrt{a}}{2 \pi} \int_{-4 / a}^{0} d y \frac{y}{\sqrt{-y^{3}-\frac{a}{4} y^{4}}}=-1
$$


If we recall that $g-g_{c}=g_{c} a^{2} \mu$, the leading non-analytic power of $g-g_{c}$ is given by the term involving the Fermi energy. Since it is different from zero for both positive and negative cosmological constants, according to (2.18) and (2.19) we conclude that the (1.2) must be violated even at the spherical level by the stochastic regularization.

If we integrate $(2.24)$ with respect to $g-g_{c}$ we get

$$
<\mathcal{O}_{0}>_{F P}=\frac{\lambda_{c}}{2}\left(c_{0}+c_{2} \mu a^{2}-e_{f} a^{3}+O\left((\sqrt{|\mu|} a)^{7 / 2}\right)\right)
$$

and from (2.24) and (2.25) it is clear that the leading non-analyticity in $\mu$ (or $g-g_{c}$ ) is entirely contained in the Fermi energy $e_{f}$ which therefore can be considered as the puncture operator ${ }^{4}$.

Let us end this section with a discussion of the rôle of relations like (2.20): $<\mathcal{O}_{0}>=-\Gamma^{\prime}(g) / N^{2}$ in the context of stochastic quantization. Due to the hidden supersymmetry, which is unbroken at the spherical level for $\mu>0$ it is clear that such a relation can not be true if we for $\Gamma$ use the energy of the $N$-Fermi system, since the total energy of the $N$-Fermi system is exactly zero for all $\mu>0$. However, it is possible to have a somewhat similar formula in the stochastic scheme. By definition we have from the second equality in (2.20) that $<\mathcal{O}_{0}>_{F P}=<\operatorname{Tr} \phi / N>_{F P}$ and we can get this quantity from the free energy $\Gamma_{F P}$ of the $N$-Fermi system if we add a source term $J \operatorname{Tr} \phi / N$ to the FP-hamiltonian. This term explicitly breaks supersymmetry and $\Gamma_{F P}(J)$ will be different from zero and we have, as noticed in $[6]$ :

$$
<\mathcal{O}_{0}>_{F P}=\left.\frac{d \Gamma_{F P}(J, g)}{d J}\right|_{J=0}
$$

The modified single particle hamiltonian will be

$$
h_{f p}=-4 \frac{d^{2}}{d y^{2}}+v_{f p}(y, \mu, j, a)
$$

where $j$ is an appropriately scaled source term and

$$
v_{f p}(y, \mu, j, a)=-(3 \mu+j) y+y^{3}+\left[-\frac{3}{2} \mu a y^{3}+\frac{a}{4} y^{4}\right]
$$

4 This identification of the Fermi energy as the puncture operator is only valid for the simplest potentials $V(\phi)$ which allow a factorization of the ground state $\Psi_{0}$ in single particle wave functions [18]. 
It is now clear that taking the limit $a \rightarrow 0$ before calculating expectation values means that we are breaking supersymmetry in a way identical to adding a source term $j$ since only the combination $3 \mu+j$ appears in (2.28) after $a$ is zero. Of course the integrals defining the expectation values will now be divergent since we have dropped the stabilizing term in the potential. However, if one just introduces a cut-off $\Lambda \sim-1 / a$ in the integrals it can be shown that the leading non-analytical behaviour is still the same as with the full potential. In this way one can convert (2.26) to an equation similar to (2.20) where the differentiation is with respect to $\mu$ and where $\Gamma_{F P}(\mu)$ is the energy calculated from the $N$-Fermion system with the unbounded potential $-3 \mu y+y^{3}$, but with a lower cut-off $\Lambda \sim-1 / a$. All results in this section can be derived using this technique.

\section{Macroscopic Loops on the Sphere.}

Motivated by ref.[15], we make some remarks on the expectation value of a single macroscopic loop in the stochastic quantization scheme. We only consider the spherical limit, however as we have seen above the behaviour of the spherical limit for $\mu<0$ is in a sense non-perturbative. It is not unreasonable to expect the full non-perturbative result to have a similar qualitative (but more smoothed out) behaviour.

From identification through the (dual of the) Feynman diagram expansion in the hermitian matrix model we know that the operator representing the insertion of an $n$ - polygonal loop is given by

$$
<W(n)>=<\frac{1}{N} \operatorname{Tr} \phi^{n}>
$$

This class of observables belongs to the ones which can readily be defined in the stochastic regularization scheme. In the spherical approximation (2.5) we have

$$
<W(n)>=\frac{1}{\pi} \int_{\lambda_{r}}^{\lambda_{l}} d \lambda \sqrt{E_{F}-V_{F P}(\lambda)} \lambda^{n} .
$$

For finite $n$ each observable clearly has the same critical behaviour as the puncture operator. However interesting behaviour arises if one scales $n \rightarrow \infty$ as

$$
n=l / a
$$


In this way we can identify $l$ with a macroscopic length of the loop, $a$ being identified with the "lattice" spacing. Introducing the scaled variables (2.9) in (3.2) we get

$$
<W\left(\frac{l}{a}\right)>=\frac{\lambda_{c}^{l / a}}{N} \frac{1}{2 \pi} \int_{y_{r}}^{y_{l}} d y \sqrt{e_{f}-v_{f p}(y)}(1+a y)^{l / a}
$$

In the scaling limit $(1+a y)^{l / a} \rightarrow e^{l y}$ and we get

$$
<W\left(\frac{l}{a}\right)>=\frac{\lambda_{c}^{l / a}}{N}<w(l)>; \quad<w(l)>\equiv \frac{1}{2 \pi} \int_{y_{r}}^{y_{l}} d y \sqrt{e_{f}-v_{f p}(y)} \mathrm{e}^{l y} .
$$

This expression is perfectly well defined for both positive and negative $\mu$ as long as $l$ is positive. For $l$ negative (3.1)-(3.4) are not well defined and this is reflected in the exponential divergence of (3.5) for $a \rightarrow 0$. As is well known [13][19] [20] the Laplace transform of $w(l)$ is the generator of one point functions and has very transparent analyticity properties. It is defined by

$$
F(z)=\int_{0}^{\infty} d l \mathrm{e}^{-l z}<w(l)>=\frac{1}{2 \pi} \int_{y_{l}}^{y_{r}} d y \frac{\sqrt{e_{f}-v_{f p}(y)}}{y-z}
$$

From this integral representation it is clear that $F(z)$ is analytic everywhere in the complex plane except along the cut $z \in\left[y_{l}, y_{r}\right]$ and we can express $\langle w(l)\rangle$ by the inverse laplace transform:

$$
<w(l)>=\int_{-i \infty+r}^{i \infty+r} \frac{d z}{2 \pi i} \mathrm{e}^{z l} F(z)
$$

Here $r$ is to the right of any singularities of $F(z)$ in the $z$ complex plane, i.e. to the right of $y_{r}$. In fact (3.7) makes sense even for negative $l$ and can be considered as a definition of negative length loops. For $l$ negative we can clearly close the contour to the right in (3.7) and we get

$$
<w(l)>=0 \quad \text { for } \quad l<0
$$

This physically very reasonable result is true independently of whether $\mu$ is positive or negative ${ }^{5}$.

5 We should note that our definition seems to differ from the one given in [15] where one gets an expectation value of $w(l)$ for $l<0$. 
Up to now the there has been no difference between negative and positive cosmological constants $\mu$. However the cut-structures of $2 \pi u_{f p}(z) \equiv \sqrt{e_{f}-v_{f p}(z)}$ in the complex plane are different. The hidden supersymmetry, unbroken for $\mu>0$, ensure that in this case $e_{f}-v_{f p}(z)$ has a double zero $y_{0}=\sqrt{|\mu|}+O(a)$ and in this case (recall that $y_{l}=-4 / a+O(a)$ ):

$$
2 \pi u_{f p}(z)=\left(y_{0}-z\right) \sqrt{\left(y_{r}-z\right)\left(z-y_{l}\right) a / 4} .
$$

However, for $\mu<0$ the double zero $y_{0}$ splits in two complex zeroes given approximately by

$$
z_{ \pm}=-\frac{y_{r}}{2} \pm i \frac{\sqrt{3}}{2} \sqrt{y_{r}^{2}+4|\mu|}
$$

and

$$
2 \pi u_{f p}(z)=\sqrt{\left(z-z_{+}\right)\left(z-z_{-}\right)\left(y_{r}-z\right)\left(z-y_{l}\right) a / 4}
$$

has an additional cut from $z_{+}$to $z_{-}$. From the integral representation (3.6) it is clear that $F(z)$ falls off as $1 / z$ for $|z| \rightarrow \infty$, is analytic except along the cut $\left[y_{l}, y_{r}\right]$ on the real axis and that

$$
F(y+i \varepsilon)-\left.F(y-i \varepsilon)\right|_{y \in\left(y_{l}, y_{r}\right)}=2 \pi i u(y)
$$

When $u(z)$ is given by (3.9) the cut of $u(z)$ coincide with the one of $F(z)$ and $F(z)$ is uniquely determined as

$$
F(z)=\frac{1}{2} V^{\prime}(z)+i \pi u(z)
$$

where $\frac{1}{2} V^{\prime}(z)$ is given by (2.7) and determined uniquely by the requirement that $F(z)=O(1 / z)$ for $|z| \rightarrow \infty$.

For $\mu<0$ the additional cut in $u(z)$ invalidates (3.13), and one has to subtract a function with this cut and which falls of as $1 / z$. It is uniquely determined as

$$
\frac{1}{2} X^{\prime}(z)=\frac{1}{2 \pi i} \oint_{\mathcal{C}} d z^{\prime} \frac{u\left(z^{\prime}\right)}{z^{\prime}-z}
$$

where the contour $\mathcal{C}$ surrounds the new cut. $F$ is now given as

$$
F(z)=\frac{1}{2}\left(V^{\prime}(z)+X^{\prime}(z)\right)+i \pi u(z) .
$$


From the classic work [21] of Brezin et al. it is known that if the eigenvalue distribution of the 0D-matrix model in the large $N$ limit is given by $u(y)$, defined on an interval $\left[y_{l}, y_{r}\right]$, then the resolvent $F(z)$, defined by ${ }^{6}$

$$
F(z)=\int_{y_{l}}^{y_{r}} d y \frac{u(y)}{y-z}
$$

is related to the potential $V_{\text {eff }}(\phi)$ of the matrix model by

$$
F(y \pm i \epsilon)=\frac{1}{2} V^{\prime}{ }_{e f f}(y) \pm i \pi u(y), \quad y \in\left[y_{l}, y_{r}\right] .
$$

From (3.13) and (3.15) we conclude that the 0D matrix model effective potential which gives the same results as the FP-potential in the spherical limit is given by

$$
V_{e f f}=V+\theta(-\mu) X
$$

Note that $X$ is non-polynomial. It would be interesting to examine whether the full stochastic quantization scheme solution would also follow from $V_{\text {eff }}$.

\section{A Numerical Comparison.}

We have shown analytically in sects. 1 and 2 that the stochastic quantization scheme is different from the KdV flow method, by comparing their leading asymptotics as $\mu \rightarrow-\infty$. It is of interest to compare them in the small $\mu$ regime too. We can do this numerically using the one-point function for the puncture operator, $\left\langle\mathcal{O}_{0}\right\rangle$, calculated in ref.[6] in the stochastic quantization scheme and $\left\langle\mathcal{O}_{0}\right\rangle$ calculated in the KdV flow method in ref.[9].

We refer the reader to these papers for more details on those calculations. In ref.[6] the real and imaginary parts of the non-perturbative hermitian matrix model solution are also computed and plotted. We have copied the five points for the stochastic quantization scheme (the circles) and for the real part of the hermitian

6 We have the relation

$$
R(w) \equiv \frac{1}{N}<\operatorname{Tr} \frac{1}{\phi-w}>=\frac{a^{3 / 2}}{\lambda_{c}} F(z), \quad w=\lambda_{c}(1+a z)
$$

where $R(w)$ denotes the resolvent in unscaled variables. 
matrix model solution (the triangles) onto fig.1, using the conventions of ref.[6]. The remaining line in fig. 1 is calculated in the $\mathrm{KdV}$ flow method[9]. The errors in this line are negligable $\left(\sim 10^{-5}\right)$. Although the three graphs clearly differ, it is intriguing that they are qualitatively so similar.

The translation from the conventions of ref.[9] to those of ref.[6] is as follows:

$$
P-P_{0}=8 \sqrt{3} \mu^{-3 / 2}\left(<\mathcal{O}_{0}>-\frac{2}{3} \mu^{3 / 2}\right)
$$

is the sphere subtracted one-point function, up to factors as indicated, and is called $\mathcal{F}$ and $\mathcal{G}$ in ref.[6]. The horizontal coordinate $z$ is proportional to the cosmological constant: $z=\frac{1}{4}(2 \sqrt{3})^{2 / 5} \mu$. These translations are derived using the recursion relations of the orthogonal polynomial coefficients $\tilde{r}_{n}$ and $\tilde{a}_{n}$ (eqns.(26) and (27) of ref.[6]) to calculate the spherical contribution of ref.[6]'s definition of the one point function

$$
<\mathcal{O}_{0}>\equiv<\frac{1}{N} \operatorname{Tr} \varphi^{2}>=\frac{1}{3 g N} \sum_{n=0}^{N-1} \tilde{a}_{n}
$$

in the contour rotated hermitian matrix model. Using the continuum limits given there in eqns. $(29-31)^{7}$ we find

$$
P=\frac{<\frac{1}{N} \operatorname{Tr} \varphi^{2}>}{\left(1-g / g_{c}\right)^{3 / 2}}=-8 \sqrt{3} \int_{1}^{\infty} d y f(y)
$$

where $f$ satisfies the Painlevé I equation in the form

$$
\frac{\sqrt{3}}{48 z^{5 / 2}} \frac{d^{2} f}{d y^{2}}+f^{2}=y
$$

We use this and eqn.(1.4) to make the fundamental identifications $f=-\rho(x) / \sqrt{\mu}$, $y=x / \mu$ and $z=\frac{1}{4}(2 \sqrt{3})^{2 / 5} \mu$ from which it follows that (4.1) translates to

$$
P=\frac{8 \sqrt{3}}{\mu^{3 / 2}} \int_{\mu}^{\infty} d x \rho(x)
$$

\footnotetext{
7 Eqn.(30) should read $a^{2} y_{n}=\cdots$
} 


\section{Conclusions}

As shown above the KdV flow symmetry of $2 \mathrm{D}$ quantum gravity is broken non-perturbatively by the real solution provided by stochastic quantization scheme. Since another scheme exists which preserves these flows non-perturbatively and which allows for a real solution [9], and since we have found no obvious unphysical behaviour of physical observables in the two schemes (as discussed in sec. 3), one is left with ambiguities. On the one hand we have the general method of stochastic stabilization of bottomless actions [1], which works in any dimension. As already pointed out in ref.[6] it disagrees with the stabilization via contour rotation [22]

. In $2 \mathrm{~d}$ gravity the contour rotation method satisfy the KdV-flow symmetry, but leads to unacceptable complex solutions. On the other hand the unique real solution to (1.2) is an alternative candidate for a non-perturbative definition of $2 \mathrm{~d}$ gravity[9]. The preference of this definition to the one of stochastic stabilization would amount to the declaration that $\mathrm{KdV}$-flows are a fundamental property of nonperturbative $2 \mathrm{D}$ quantum gravity. Keeping in mind on the one hand the ultimate goals of understanding higher dimensional gravity and string theory, and on the other, the lack of understanding of the fundamental physics behind the KdV-flow structure, it seems to us rather that the results obtained in this article highlight the need for a better understanding of the basic principles underlying non-perturbative quantum gravity.

\section{Acknowledgements}

TRM would like to thank the Neils Bohr Institute for hospitality during the first stages of this work, and Simon Dalley for helpful comments. CVJ thanks the S.E.R.C. for financial support and JA thanks Charlotte F. Kristjansen and Jens Lyng Petersen for helpful discussions. 


\section{References}

[1] J. Greensite and M. Halpern, Nucl. Phys. B242 (1984) 167.

[2] E.Marinari and G.Parisi, Phys. Lett. 240B (1990) 375.

[3] E.Marinari and G.Parisi, Phys. Lett. 247B (1990) 537.

[4] J.Ambjørn, J.Greensite and S.Varsted, Phys. Lett. 249B (1990) 411.

[5] M.Karliner and A.Migdal, Mod. Phys. Lett. 5A (1990) 2565.

[6] J.Ambjørn and J.Greensite, Phys. Lett. B254 (1991) 66.

[7] J.González and M.A.H. Vozmediano, Instituto de Estructura de la Materia preprint IEM-FT-35/90.

[8] S.Dalley, C.Johnson and T.Morris, Southampton Preprint SHEP 90/91-16.

[9] S.Dalley, C.Johnson and T.Morris, Southampton Preprint SHEP 90/91-28.

[10] D.J. Gross and A.A. Migdal, Nucl. Phys. B340 (1990) 333.

[11] T.Banks, M.Douglas, N.Seiberg and S.Shenker, Phys. Lett. 238B (1990) 279.

[12] E.Brézin and V.Kazakov, Phys. Lett. B236 (1990) 144;

M.Douglas and S.Shenker, Nucl. Phys. B335 (1990) 135;

D.Gross and A.A.Migdal, Phys. Rev. Lett. 64 (1990) 127.

[13] F.David, Mod. Phys. Lett. A5 (1990) 1019.

[14] F.David Nucl. Phys. B348 (1991) 507.

[15] F.David, Notes added to talk given at the Cargese Workshop "Random Surfaces, Quantum Gravity and Strings", May 28-June 1, 1990.

[16] I.R.Gelfand and L.A.Dikii, Russian Math. Surveys 30 (1975) 77.

[17] S.Dalley, C.Johnson and T.Morris, Southampton Preprint SHEP-90/91-35, talk given at Barcelona Workshop "Random Surfaces and 2D Quantum Gravity", 10-14 June 1991 to appear in Nucl. Phys. B Proc. Suppl.;

C.Johnson, T.Morris and B.Spence, in preparation.

[18] J. Ambjørn and C.F. Kristjansen, to appear

[19] J. Ambjørn and Y. Makeenko, Mod. Phys. Lett. A5 (1990) 1753

[20] J. Ambjørn, J. Jurkiewicz and Y. Makeenko, Phys.Lett. 251B (1990) 517

[21] E. Brezin, C. Itzykson, G. Parisi and J.B Zuber, Commun.math.Phys. 59, (1978) 35 
[22] K. Gawedzi and A. Kupiainen, Nucl. Phys. B257[FS14] (1985) 474;

G. Gibbons, S. Hawking, and M. Perry, Nucl. Phys. B138 (1978) 141. 


\section{Figure Captions}

Fig. 1: The expectation value of the puncture operator with the sphere contribution removed; $z$ is proportional to the cosmological constant. The triangles are the real part of the hermitian matrix model result. The circles are values given by the stochastic quantization scheme, and the unmarked line is the result of the $\mathrm{KdV}$ flow scheme. 